\title{
Prevalência de morbidades e sintomas em idosos: um estudo comparativo entre zonas rural e urbana
}

\author{
The prevalence of morbidity and symptoms among the elderly: \\ a comparative study between rural and urban areas
}

Eveline Fronza da Silva ${ }^{1}$

Vera Maria Vieira Paniz ${ }^{2}$

Gabriela Laste ${ }^{3}$

Iraci Lucena da Silva Torres ${ }^{3}$

\footnotetext{
${ }^{1}$ Universidade Luterna do Brasil. Av. Farroupilha 8001, São José. 92425-900 Canoas RS.

evelinefronza@yahoo.com.br ${ }^{2}$ Programa de Pós-Graduação em Saúde Coletiva, Centro de Ciências da Saúde, Universidade do Vale do Rio dos Sinos.

${ }^{3}$ Instituto de Ciências Básicas da Saúde, Universidade Federal do Rio Grande do Sul.
}

\begin{abstract}
A cross-sectional study was conducted with the elderly population in rural and urban areas characterized by the prevalence of morbidity and symptoms, and 229 elderly people were interviewed. The average age was 72.3, of which $57.2 \%$ lived in the rural zone and $56.3 \%$ were female. The morbidities most reported were insomnia (37.7\%), anxiety (32.1\%), depression $(26.7 \%)$, and in the rural zone it was diabetes (13.3\%). In this zone, Alzheimer's disease was more prevalent among the elderly who handled pesticides $(21.7 \%)$. The most prevalent symptoms among urban zone residents were: cough/runny nose and sight alterations (41.2\%), allergy/itching (11.4\%). In the rural zone, dry mouth (25.4\%), sight alterations (35.6\%) and leg pain (66.1\%) were also more prevalent among those who used pesticides. The inadequate use of Individual Protection Equipment was $85.4 \%$, and $45.1 \%$ also disposed of pesticide packaging inappropriately. The setting up of public health programs is necessary to promote health among the elderly and the potential exposure to pesticides for this population should be seen as a health risk determinant. Key words Elderly, Health of the elderly, Pesticides, Rural population, Cross-sectional studies
\end{abstract}

Resumo Realizou-se um estudo transversal, com a população idosa moradora nas zonas rural e urbana caracterizando a prevalência de morbidades e sintomas. Entrevistou-se 229 idosos, com média de idade de 72,3 anos, 57,2\% residem na zona urbana, 56,3\% são do sexo feminino. As morbidades mais relatadas por residentes da zona urbana foram: insônia (37,7\%), ansiedade (32,1\%), depressão (26,7\%); e na rural, diabetes (13,3\%). Nesta, a Doença de Alzheimer foi mais prevalente em idosos que utilizam agrotóxicos $(21,7 \%)$. Os sintomas mais prevalentes por moradores da zona urbana foram: tosse/coriza e visão alterada (41,2\%), alergia/coceira (11,4\%). Na rural, boca seca $(25,4 \%)$, visão alterada $(35,6 \%)$ e dor nas pernas $(66,1 \%)$ que também foram mais prevalentes em quem utilizava agrotóxicos. O uso de Equipamentos de Proteção Individual de forma incompleta foi de $85,4 \%$ e 45,1\% descartam as embalagens de forma inadequada. Há a necessidade de desenvolvimento de programas de saúde pública que auxiliem na promoção de saúde do idoso, e que considere a possivel exposição dessa população a agrotóxicos com um determinante em saúde.

Palavras-chave Idoso, Saúde do idoso, Praguicidas, População rural, Estudos transversais 


\section{Introdução}

O Brasil está em crescente envelhecimento populacional, resultante da redução das taxas de natalidade que vem ocorrendo desde os anos 60, e a diminuição progressiva das taxas de mortalidade, a partir do final da Segunda Guerra Mundial nos anos $40^{1-3}$. Estima-se que em 2050, haverá 226 idosos de 60 anos ou mais para cada 100 crianças e adolescentes ${ }^{4}$.

Com a mudança no perfil de saúde da população, tornam-se predominantes doenças crônicas e suas complicações, que resultam em maior utilização de serviços de saúde, em relação aos processos agudos que são resolvidos rapidamente, seja pela cura ou por óbito. As enfermidades relacionadas a problemas do aparelho circulatório são as que mais atingem as pessoas na faixa de 60 anos ou mais ${ }^{3}$. Além destas, doenças cardiovasculares (infarto, angina, insuficiência cardíaca), acidente vascular encefálico, câncer, pneumonia, enfisema e bronquite crônica, infecção urinária, diabetes, osteoporose, osteartrose também acometem frequentemente os idosos ${ }^{5}$.

No processo de envelhecimento é comum a ocorrência de doenças neurodegenerativas, como Doença de Parkinson e Doença de Alzheimer que afetam a funcionalidade das pessoas idosas. Estudos demonstram que a dependência para o desempenho das atividades de vida diária, tende a aumentar cerca de 5\% na faixa etária de 60 anos, para cerca de $50 \%$ entre aqueles com 90 ou mais anos, esses dados são ainda maiores na presença das doenças neurodegenerativas ${ }^{6}$.

Além do aumento da idade, outros fatores podem contribuir para o surgimento de doenças crônicas, como fatores ambientais e exposição a produtos tóxicos. Segundo a literatura, dentre os fatores ambientais que podem estar relacionados à prevalência de doenças e de sintomas em idosos destaca-se a exposição a agrotóxicos ${ }^{7}$.

Devido ao aumento da demanda de alimentos, o setor de agricultura, passa por um período de modernização que, apesar de gerar crescimento econômico, apresenta riscos potenciais ao ambiente e à saúde humana. $\mathrm{O}$ uso indiscriminado desses produtos contribui para a contaminação ambiental e para o aumento das intoxicações, principalmente ocupacionais ${ }^{8}$. De acordo com a classe química pertencente a esses produtos e o tipo de exposição, podem causar dermatites, cânceres e sintomas neuropsiquiátricos ${ }^{9,10}$.

Há relatos de que indivíduos expostos a esses agentes por vários anos, por ação cumulativa, possam apresentar alterações na funcionalidade de diversos sistemas do organismo, dependendo do metabolismo do indivíduo, bem como das enzimas envolvidas nos processos metabólicos ${ }^{10,11}$.

Considerando o aumento da população idosa e a possibilidade desta ter sido exposta ou ter tido contato com agrotóxicos, este estudo objetivou caracterizar a população de idosos que reside no município de Cachoeira do Sul (RS), investigando morbidades e sintomas referidos segundo zona de moradia rural/urbana e uso de agrotóxicos, descrevendo ainda, as características do uso destes produtos.

\section{Métodos}

Realizou-se um estudo transversal na população de idosos com 60 anos ou mais, residentes nas zonas urbana e rural do município de Cachoeira do Sul (RS). Este, localizado na região do JacuíCentro no sul do Brasil, caracteriza-se por uma economia essencialmente agrária, destacando-se as lavouras de arroz com 37.270 ha de área plantada, milho com 5.000 ha, soja com 48.000 ha e diversas cabanhas presentes no interior do município. Possui uma população, estimada no ano de 2010, de 83.827 habitantes e estima-se que $12 \%$ da população do município estejam com mais de 65 $\operatorname{anos}^{12}$.

Foi utilizada uma amostra de conveniência baseada nos setores visitados pelos censores do IBGE, no período de julho a outubro de 2007. Para o cálculo do tamanho da amostra, considerou-se uma prevalência de uso de agrotóxicos de $45 \%$, com erro de $15 \%$ e um acréscimo de $10 \%$ para possíveis perdas, estimando-se que aproximadamente 224 idosos deveriam ser entrevistados.

O trabalho de campo foi realizado pela própria pesquisadora no momento da coleta de dados da Contagem da População (2007), tanto na zona urbana quanto na rural, até contemplar a amostra. Realizou-se entrevista domiciliar utilizando um questionário estruturado e previamente testado em pesquisa anterior ${ }^{13}$. Para garantir a privacidade, a minimização de riscos e desconfortos e a busca de benefícios, todos os participantes que concordaram em participar do estudo assinaram o Termo de Consentimento Livre e Esclarecido (TCLE), ou em caso de impossibilidade do idoso responder o questionário, este era respondido pelo cuidador ou responsável que também assinava o TCLE.

As variáveis demográficas avaliadas foram: sexo; idade (em anos completos) e local de moradia (urbana/rural). Foram medidas como va- 
riáveis socioeconômicas: escolaridade (em anos completos de estudo) e ocupação (agropecuária ou outra atividade basicamente urbana). Como variáveis comportamentais foram avaliadas: consumo de tabaco (nunca fumou, ex-fumante e fumante) e consumo de bebida alcoólica (nunca consumiu, não consome mais, consome). Investigaram-se através de autorrelato as morbidades: hipertensão, insônia, ansiedade, depressão, cefaleia, diabetes, enxaqueca, hipercolesterolemia, hipertrigliceridemia, reumatismo, labirintite, Doença de Parkinson e Doença de Alzheimer. Pesquisou-se ainda, o relato dos sintomas: tosse/coriza, boca seca, visão alterada, dor lombar, dor de estômago, vômito, alergia/coceira, tremor, esquecimento, dor nas pernas, edema, náuseas, palpitação, lacrimejamento, diarreia e constipação. Avaliou-se também, o uso atual de agrotóxicos mediante a questão: "Usa algum tipo de defensivo agrícola no controle de pragas, ou algum tipo de agrotóxico?" e características da sua utilização: principal produção (pecuária e/ ou agricultura); tipo de plantação (milho, fumo, soja, arroz, consumo familiar e policultura); tipo de agrotóxico utilizado (organofosforado, carbamato, piretróide e outros); classificação do agrotóxico de acordo com sua toxicidade: faixa vermelha (classe I) - extremamente tóxico; faixa amarela (classe II) - altamente tóxico; faixa azul (classe III) - medianamente tóxico e faixa verde (classe IV) - pouco tóxico. Quanto ao preparo do agrotóxico avaliou-se: quem prepara (a própria pessoa, familiar do idoso, outra pessoa); como é preparado (dissolvido em água, podendo ser um produto concentrado ou em pó para diluir ou direto quando o produto vem pronto em pó ou já dissolvido, sem necessidade de preparo); local do preparo (pátio da casa, lavoura ou outro local) e se o utensílio de preparo é usado para outros fins. Investigou-se a forma de aplicação (a favor do vento, na ausência do vento ou independente do vento); uso de Equipamentos de Proteção Individual (EPI) durante o preparo e aplicação (completo ou incompleto) e período de aplicação (manhã, tarde ou qualquer período). Também foi avaliada a conduta no descarte da embalagem vazia, sendo classificada como conduta adequada, quando devolvida à empresa que forneceu o produto, e conduta inadequada quando fosse armazenada, queimada, depositada no subsolo, ou permanece na lavoura sem qualquer proteção.

Os dados foram digitados utilizando-se o programa Epi-Info versão 3.5.1, com checagem automática de consistência e dupla digitação, para correção de possíveis erros de digitação. A análise dos dados foi realizada no programa estatístico Stata 9.0.

A análise descritiva mediante frequência absoluta e relativa caracterizou a população de idosos geral e estratificada por zona de moradia, conforme variáveis demográficas, socioeconômicas, comportamentais e uso de agrotóxicos. As morbidades e os sintomas referidos pelos idosos foram descritos segundo a zona de moradia e uso de agrotóxicos. As características relacionadas ao uso de agrotóxico com respectivas proporções também foram detalhadas. Para testar as diferenças entre proporções, utilizou-se o teste do qui-quadrado ou teste exato de Fisher quando o primeiro não preenchia os pressupostos.

O protocolo segue as condições estabelecidas na Resolução 196/96 do Conselho Nacional de Saúde $(\mathrm{CNS})^{14}$ e foi aprovado pelo Comitê de Ética da Universidade do Vale do Rio dos Sinos.

\section{Resultados}

Dentro do período selecionado para a pesquisa, entrevistou-se 229 indivíduos na faixa etária estudada. A média de idade da amostra foi de 72,3 anos $(\mathrm{dp}=7,7)$ com amplitude de 60 a 97 anos, sendo que $57,2 \%$ dos idosos entrevistados residiam na zona urbana.

A Tabela 1 descreve as características da população estudada quanto às variáveis demográficas, socioeconômicas, comportamentais e uso de agrotóxicos, estratificada por zona de moradia. Entre os idosos, 56,3\% eram do sexo feminino e $35,9 \%$ tinham 75 anos ou mais de idade. Com relação à escolaridade, entre idosos moradores da zona rural, $63,9 \%$ possuíam até quatro anos completos de estudo e $18,6 \%$ nunca tinham frequentado a escola; enquanto que para os idosos da zona urbana esses percentuais foram de $33,6 \%$ e $13,3 \%$, respectivamente $(\mathrm{p}<0,001)$. Quanto à ocupação, as atividades ligadas à agropecuária foram mais prevalentes na zona rural do que na zona urbana $(95,9 \%$ vs $10 \%$; p < $0,001)$. Na população estudada, $14,0 \%$ eram fumantes e $19,2 \%$ consumiam algum tipo de bebida alcoólica.

A prevalência de uso de agrotóxicos foi de $35,8 \%$ (IC 95\% 29,6 - 42,1), sendo significativamente maior $(\mathrm{p}<0,001)$ entre idosos moradores da zona rural 60,2\% (IC95\% 50,3-70,1) quando comparados com aqueles residentes da zona urbana 17,6\% (IC95\% 11,0 - 24,2) (Tabela 1). Entre as morbidades avaliadas, as maiores pre- 
Tabela 1. Características da população estudada conforme variáveis demográficas, socioeconômicas, comportamentais e uso de agrotóxicos. Cachoeira do Sul, RS, Brasil, 2007. $(\mathrm{n}=229)$.

\begin{tabular}{|c|c|c|c|c|}
\hline \multirow[b]{2}{*}{ Características } & \multirow[b]{2}{*}{ N (\%) } & \multicolumn{2}{|c|}{ Zona de moradia } & \multirow[b]{2}{*}{ Valor $p^{*}$} \\
\hline & & $\begin{array}{l}\text { Rural } \\
\text { N (\%) }\end{array}$ & $\begin{array}{c}\text { Urbana } \\
\text { N (\%) }\end{array}$ & \\
\hline Total & $229(100)$ & $98(42,8)$ & $131(57,2)$ & \\
\hline Sexo & & & & 0,956 \\
\hline Masculino & $100(43,7)$ & $43(43,9)$ & $57(43,5)$ & \\
\hline Feminino & $129(56,3)$ & $55(56,1)$ & $74(56,5)$ & \\
\hline Faixa etária (anos completos) & & & & 0,084 \\
\hline $60-64$ & $27(11,9)$ & $8(8,2)$ & $19(14,7)$ & \\
\hline $65-69$ & $75(33,2)$ & $38(39,2)$ & $37(28,7)$ & \\
\hline $70-74$ & $43(19,0)$ & $22(22,7)$ & $21(16,3)$ & \\
\hline $75-97$ & $81(35,9)$ & $29(29,9)$ & $52(40,3)$ & \\
\hline Escolaridade (anos de estudo) & & & & $<0,001$ \\
\hline 0 & $35(15,6)$ & $18(18,6)$ & $17(13,3)$ & \\
\hline $1-4$ & $105(46,7)$ & $62(63,9)$ & $43(33,6)$ & \\
\hline $5-8$ & $37(16,4)$ & $11(11,3)$ & $26(20,3)$ & \\
\hline $9 \mathrm{ou}+$ & $48(21,3)$ & $6(6,2)$ & $42(32,8)$ & \\
\hline Ocupação & & & & $<0,001$ \\
\hline Atividade agropecuária & $107(46,9)$ & $94(95,9)$ & $13(10,0)$ & \\
\hline Outra & $121(53,1)$ & $4(4,1)$ & $117(90,0)$ & \\
\hline Consumo de tabaco & & & & 0,760 \\
\hline Nunca fumou & $141(61,6)$ & $63(64,3)$ & $78(59,5)$ & \\
\hline Ex-fumante & $56(24,4)$ & $22(22,4)$ & $34(26,0)$ & \\
\hline Fumante & $32(14,0)$ & $13(13,3)$ & $19(14,5)$ & \\
\hline Consumo de bebida alcoólica & & & & 0,517 \\
\hline Nunca consumiu & $148(64,6)$ & $67(68,4)$ & $81(61,8)$ & \\
\hline Não consome mais & $37(16,2)$ & $13(13,3)$ & $24(18,3)$ & \\
\hline Consome & $44(19,2)$ & $18(18,4)$ & $26(19,8)$ & \\
\hline Uso de agrotóxicos & & & & $<0,001$ \\
\hline $\operatorname{Sim}$ & $82(35,8)$ & $59(60,2)$ & $23(17,6)$ & \\
\hline Não & $147(64,2)$ & $39(39,8)$ & $108(82,4)$ & \\
\hline
\end{tabular}

O número máximo de valores ignorados foi 4 para a variável escolaridade. ${ }^{*}$ Valor $p$ do teste do qui-quadrado para heterogeneidade de proporções.

valências foram observadas para hipertensão $(52 \%)$ e insônia $(31,1 \%)$, seguidas de ansiedade $(22,3 \%)$, reumatismo $(19,6 \%)$ e depressão $(18,3 \%)$.

A Tabela 2 apresenta prevalências das morbidades referidas pelos idosos, estratificada por zona de moradia e por uso de agrotóxicos. Observou-se que o relato de algumas morbidades foi estatisticamente diferente entre zonas de moradia, sendo estas mais prevalentes na zona urbana do que na rural. São elas: insônia $(37,7 \%$ vs $22,4 \%$; $\mathrm{p}=0,014)$, ansiedade $(32,1 \%$ vs $9,2 \% \mathrm{p}<$ $0,001)$, depressão $(26,7 \%$ vs $7,1 \%$; $p<0,001)$, cefaleia $(22,9 \%$ vs $2,0 \%$; p < 0,001), Doença de Parkinson (9,9\% vs 0,0\%; $\mathrm{p}=0,001)$ e Doença de Alzheimer ( $8,4 \%$ vs 0,$0 ; p=0,003)$. Situação contrária ocorre em relação ao diabetes, o qual apre- sentou maior prevalência entre os moradores da zona rural do que nos da zona urbana $(13,3 \%$ vs $4,6 \% ; \mathrm{p}=0,018)$. A Tabela 2 mostra ainda que Doença de Alzheimer foi referida apenas por idosos residentes na zona urbana sendo nesta mais prevalente entre aqueles que utilizam agrotóxicos quando comparados com os que não utiliza$\operatorname{ram}(21,7 \%$ vs $5,6 \% ; \mathrm{p}=0,024)$.

Com relação aos sintomas avaliados, observaram-se maiores prevalências para o relato de dor nas pernas $(52,8 \%)$ e dor lombar $(52,4 \%)$, seguidos de visão alterada $(35,4 \%)$, tosse/coriza $(35,1 \%)$ e esquecimento $(32,7 \%)$. Percebeu-se ainda que cerca de $1 / 4$ dos idosos relataram edema $(27,5 \%)$ e dor de estômago $(24,9 \%)$.

A Tabela 3 apresenta as prevalências dos sintomas referidos pelos idosos, segundo zona de 
moradia rural e urbana, e uso de agrotóxicos. Salienta-se que o relato de alguns sintomas apresentou prevalências significativamente diferentes entre as zonas de moradia, sendo mais citados na zona urbana, são eles: tosse/coriza ( $41,2 \%$ vs $28,6 \% ; \mathrm{p}=0,048)$, visão alterada $(41,2 \%$ vs $27,5 \%$; $\mathrm{p}=0,032)$, alergia/coceira $(11,4 \%$ vs $4,1 \% ; \mathrm{p}=$ $0,045)$, tremor $(16,8 \%$ vs $4,1 \%$; $\mathrm{p}=0,003)$, palpitação $(19,1 \%$ vs $7,1 \%$; $=0,010)$, lacrimejamento $(8,4 \%$ vs $2,0 \% ; p=0,040)$, diarreia $(4,6 \%$ vs 0 ; $\mathrm{p}=0,031)$ e constipação $(13,7 \%$ vs $4,1 \% ; \mathrm{p}=$ 0,014).

Tabela 2. Morbidades referidas pelos idosos segundo zona de moradia rural $(n=98)$ e urbana $(n=131)$ geral e estratificada pelo uso de agrotóxicos. Cachoeira do Sul, RS, Brasil, 2007.

\begin{tabular}{|c|c|c|c|c|c|c|c|c|c|}
\hline \multirow[b]{3}{*}{ Morbidades ${ }^{a}$} & \multicolumn{4}{|c|}{ Zona Rural } & \multicolumn{4}{|c|}{ Zona Urbana } & \multirow{3}{*}{$\begin{array}{c}\text { Valor } p^{\star} \\
\text { entre } \\
\text { zona de } \\
\text { moradia }\end{array}$} \\
\hline & \multirow[b]{2}{*}{$\begin{array}{l}\text { Geral } \\
\mathrm{N}(\%)\end{array}$} & \multicolumn{2}{|c|}{ Uso de agrotóxicos } & \multirow{2}{*}{$\begin{array}{l}\text { Valor } p^{\star *} \\
\text { uso de } \\
\text { agrotóxicos }\end{array}$} & \multirow[b]{2}{*}{$\begin{array}{r}\text { Geral } \\
\text { N (\%) }\end{array}$} & \multicolumn{2}{|c|}{ Uso de agrotóxicos } & \multirow{2}{*}{$\begin{array}{c}\text { Valor } \\
p^{\star *} \text { uso de } \\
\text { agrotóxicos }\end{array}$} & \\
\hline & & $\begin{array}{c}\text { Sim } \\
\text { N (\%) }\end{array}$ & $\begin{array}{c}\text { Não } \\
\text { N (\%) }\end{array}$ & & & $\begin{array}{c}\text { Sim } \\
\text { N (\%) }\end{array}$ & $\begin{array}{c}\text { Não } \\
\text { N (\%) }\end{array}$ & & \\
\hline & $98(100)$ & $59(60,2)$ & $39(39,8)$ & & $131(100)$ & $23(17,6)$ & $108(82,4)$ & & \\
\hline \multicolumn{10}{|l|}{ Hipertensão } \\
\hline Sim & $56(57,1)$ & $31(52,5)$ & $25(64,1)$ & \multirow[t]{2}{*}{0,301} & $63(48,1)$ & $11(47,8)$ & $52(48,2)$ & \multirow[t]{2}{*}{0,999} & \multirow[t]{2}{*}{0,175} \\
\hline Não & $42(42,9)$ & $28(47,5)$ & $14(35,9)$ & & $68(51,9)$ & $12(52,2)$ & $56(51,8)$ & & \\
\hline \multicolumn{10}{|c|}{ 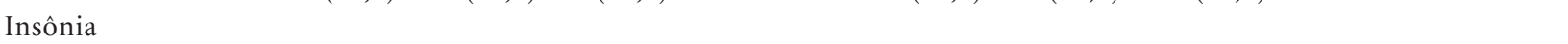 } \\
\hline Sim & $22(22,4)$ & $13(22,0)$ & $9(23,1)$ & \multirow{2}{*}{0,999} & $49(37,7)$ & $8(34,8)$ & $41(38,3)$ & \multirow[t]{2}{*}{0,816} & \multirow[t]{2}{*}{0,014} \\
\hline Não & $76(77,6)$ & $46(78,0)$ & $30(76,9)$ & & $81(62,3)$ & $15(65,2)$ & $66(61,7)$ & & \\
\hline \multicolumn{10}{|l|}{ Ansiedade } \\
\hline Sim & $9(9,2)$ & $5(8,5)$ & $4(10,3)$ & \multirow[t]{2}{*}{0,999} & $42(32,1)$ & $9(39,1)$ & $33(30,6)$ & \multirow[t]{2}{*}{0,465} & \multirow[t]{2}{*}{$<0,001$} \\
\hline Não & $89(90,8)$ & $54(91,5)$ & $35(89,8)$ & & $89(67,9)$ & $14(60,9)$ & $75(69,4)$ & & \\
\hline \multicolumn{10}{|l|}{ Depressão } \\
\hline Sim & $7(7,1)$ & $4(6,8)$ & $3(7,7)$ & 0,999 & $35(26,7)$ & $7(30,4)$ & $28(25,9)$ & 0,796 & $<0,001$ \\
\hline Não & $91(92,9)$ & $55(93,2)$ & $36(92,3)$ & 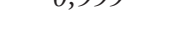 & $96(73,3)$ & $16(69,6)$ & $80(74,1)$ & & \\
\hline Cefaléia & & & & & & & & & \\
\hline Sim & $2(2,0)$ & $1(1,7)$ & $1(2,6)$ & 9 & $30(22,9)$ & $8(34,8)$ & $22(20,4)$ & 0,171 & $<0,001$ \\
\hline Não & $96(98,0)$ & $58(98,3)$ & $38(97,4)$ & S & $101(77,1)$ & $15(65,2)$ & $86(79,6)$ & & \\
\hline Diabetes & & & & & & & & & \\
\hline Sim & $13(13,3)$ & $9(15,2)$ & $4(10,3)$ & 0,555 & $6(4,6)$ & $2(8,7)$ & $4(3,7)$ & 0,284 & 0,018 \\
\hline Não & $85(86,7)$ & $50(84,8)$ & $35(89,7)$ & (0,JJנ & $125(95,4)$ & $21(91,3)$ & $104(96,3)$ & & \\
\hline Enxaque & & & & & & & & & \\
\hline Sim & $5(5,1)$ & $3(5,1)$ & $2(5,1)$ & 0 & $9(6,9)$ & 0 & $9(8,3)$ & 0,359 & 0,581 \\
\hline Não & $93(94,9)$ & $56(94,9)$ & $37(94,9)$ & 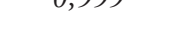 & $122(93,1)$ & $23(100)$ & $99(91,7)$ & & \\
\hline Hipercolesterolemi & & & & & & & & & \\
\hline Sim & $11(11,2)$ & $5(8,5)$ & $6(15,4)$ & 0,337 & $22(16,8)$ & $3(13,0)$ & $19(17,6)$ & 0,764 & 0,235 \\
\hline Não & $87(88,8)$ & $54(91,5)$ & $33(84,6)$ & ( & $109(83,2)$ & $20(87,0)$ & $89(82,4)$ & & \\
\hline Hipertrigliceridemi & & & & & & & & & \\
\hline Sim & $4(4,1)$ & $2(3,4)$ & $2(5,1)$ & & $6(4,6)$ & 0 & $6(5,6)$ & 0,590 & 0,855 \\
\hline Não & $94(95,9)$ & $57(96,6)$ & $37(94,9)$ & 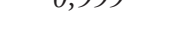 & $125(95,4)$ & $23(100)$ & $102(94,4)$ & & \\
\hline Reumatismo & & & & & & & & & \\
\hline $\operatorname{Sim}$ & $15(15,3)$ & $7(11,9)$ & $8(20,5)$ & 0,265 & $30(22,9)$ & $5(21,7)$ & $25(23,1)$ & 0,999 & 0,152 \\
\hline Não & $83(84,7)$ & $52(88,1)$ & $31(79,5)$ & 0,200 & $101(77,1)$ & $18(78,3)$ & $83(76,9)$ & & \\
\hline Labirintite & & & & & & & & & \\
\hline Sim & $8(8,2)$ & $6(10,1)$ & $2(5,1)$ & $0,4 / 1$ & $14(10,7)$ & $3(13,0)$ & $11(10,2)$ & 0,712 & 0,521 \\
\hline Não & $90(91,8)$ & $53(89,9)$ & $37(94,9)$ & 0,7 & $117(89,3)$ & $20(87,0)$ & $97(89,8)$ & & \\
\hline Doença de Parkiı & & & & & & & & & \\
\hline Sim & 0 & 0 & 0 & - & $13(9,9)$ & $4(17,4)$ & $9(8,3)$ & 0,242 & 0,001 \\
\hline Não & $98(100)$ & $59(100)$ & $39(100)$ & & $118(90,1)$ & $19(82,6)$ & $99(91,7)$ & & \\
\hline Doença de Alzhein & & & & & & & & & \\
\hline Sim & 0 & 0 & 0 & - & $11(8,4)$ & $5(21,7)$ & $6(5,6)$ & 0,024 & 0,003 \\
\hline Não & $98(100)$ & $59(100)$ & $39(100)$ & & $120(91,6)$ & $18(78,3)$ & $102(94,4)$ & & \\
\hline
\end{tabular}

a - O número máximo de valores ignorados foi de 1 para a variável “insônia” na população urbana. * valor p do teste do qui-quadrado para heterogeneidade de proporções. ${ }^{* *}$ valor p do teste exato de Fisher para heterogeneidade de proporções. 
Tabela 3. Sintomas referidos pelos idosos segundo zona de moradia rural $(\mathrm{n}=98)$ e urbana $(\mathrm{n}=131)$ geral e estratificada pelo uso de agrotóxicos. Cachoeira do Sul, RS, Brasil, 2007.

\begin{tabular}{|c|c|c|c|c|c|c|c|c|c|}
\hline \multirow[b]{3}{*}{ Sintomas a } & \multicolumn{4}{|c|}{ Zona Rural } & \multicolumn{4}{|c|}{ Zona Urbana } & \multirow{3}{*}{$\begin{array}{c}\text { Valor } p^{\star} \\
\text { entre } \\
\text { zona de } \\
\text { moradia }\end{array}$} \\
\hline & \multirow[b]{2}{*}{$\begin{array}{l}\text { Geral } \\
\text { N }(\%)\end{array}$} & \multicolumn{2}{|c|}{ Uso de agrotóxicos } & \multirow{2}{*}{$\begin{array}{c}\text { Valor } p^{\star *} \\
\text { uso de } \\
\text { agrotóxicos }\end{array}$} & \multirow[b]{2}{*}{$\begin{array}{l}\text { Geral } \\
\text { N (\%) }\end{array}$} & \multicolumn{2}{|c|}{ Uso de agrotóxicos } & \multirow{2}{*}{$\begin{array}{c}\text { Valor } \\
p^{\star *} \text { uso de } \\
\text { agrotóxicos }\end{array}$} & \\
\hline & & $\begin{array}{c}\text { Sim } \\
\text { N (\%) }\end{array}$ & $\begin{array}{c}\text { Não } \\
\text { N (\%) }\end{array}$ & & & $\begin{array}{c}\text { Sim } \\
\text { N (\%) }\end{array}$ & $\begin{array}{c}\text { Não } \\
\text { N (\%) }\end{array}$ & & \\
\hline Total & $98(100)$ & $59(60,2)$ & $39(39,8)$ & & $131(100)$ & $23(17,6)$ & $108(82,4)$ & & \\
\hline Tosse/coriza & & & & 0,820 & & & & 0,060 & 0,048 \\
\hline Sim & $28(28,6)$ & $16(27,1)$ & $12(30,8)$ & & $54(41,2)$ & $14(60,9)$ & $40(37,0)$ & & \\
\hline Não & $70(71,4)$ & $43(72,9)$ & $27(69,2)$ & & $77(58,8)$ & $9(39,1)$ & $68(63,0)$ & & \\
\hline Boca Seca & & & & $<0,001$ & & & & 0,767 & 0,548 \\
\hline Sim & $15(15,3)$ & $15(25,4)$ & 0 & & $24(18,3)$ & $5(21,7)$ & $19(17,6)$ & & \\
\hline Não & $83(84,7)$ & $44(74,6)$ & $39(100)$ & & $107(81,7)$ & $18(78,3)$ & $89(82,4)$ & & \\
\hline Visão alterada & & & & 0,038 & & & & 0,253 & 0,032 \\
\hline Sim & $27(27,5)$ & $21(35,6)$ & $6(15,4)$ & & $54(41,2)$ & $12(52,2)$ & $42(38,9)$ & & \\
\hline Não & $71(72,5)$ & $38(64,4)$ & $33(84,6)$ & & $77(58,8)$ & $11(47,8)$ & $66(61,1)$ & & \\
\hline Dor lombar & & & & 0,098 & & & & 0,823 & 0,529 \\
\hline Sim & $49(50,0)$ & $34(57,6)$ & $15(38,5)$ & & $71(54,2)$ & $13(56,5)$ & $58(53,7)$ & & \\
\hline Não & $49(50,0)$ & $25(42,4)$ & $24(61,5)$ & & $60(45,8)$ & $10(43,5)$ & $50(46,3)$ & & \\
\hline Dor de estômago & & & & 0,646 & & & & 0,785 & 0,421 \\
\hline Sim & $27(27,5)$ & $15(25,4)$ & $12(30,8)$ & & $30(22,9)$ & $6(26,1)$ & $24(22,2)$ & & \\
\hline Não & $71(72,5)$ & $44(74,6)$ & $27(69,2)$ & & $101(77,1)$ & $17(73,9)$ & $84(77,8)$ & & \\
\hline Vômito & & & & 0,516 & & & & 0,629 & 0,136 \\
\hline Sim & $2(2,0)$ & $2(3,4)$ & 0 & & $8(6,1)$ & $2(8,7)$ & $6(5,6)$ & & \\
\hline Não & $96(98)$ & $57(96,6)$ & $39(100)$ & & $123(93,9)$ & $21(91,3)$ & $102(94,4)$ & & \\
\hline Alergia/Coceira & & & & 0,999 & & & & 0,468 & 0,045 \\
\hline Sim & $4(4,1)$ & $3(5,1)$ & $1(2,6)$ & & $15(11,4)$ & $1(4,3)$ & $14(13,0)$ & & \\
\hline Não & $94(95,9)$ & $56(94,9)$ & $38(97,4)$ & & $116(88,6)$ & $22(95,7)$ & $94(87,0)$ & & \\
\hline Tremor & & & & 0,999 & & & & 0,540 & 0,003 \\
\hline Sim & $4(4,1)$ & $3(5,1)$ & $1(2,6)$ & & $22(16,8)$ & $5(21,7)$ & $17(15,7)$ & & \\
\hline Não & $94(95,9)$ & $56(94,9)$ & $38(97,4)$ & & $109(83,2)$ & $18(78,3)$ & $91(84,3)$ & & \\
\hline Esquecimento & & & & 0,015 & & & & 0,811 & 0,755 \\
\hline Sim & $31(31,6)$ & $13(22,0)$ & $18(46,1)$ & & $44(33,6)$ & $7(30,4)$ & $37(34,3)$ & & \\
\hline Não & $67(68,4)$ & $46(73,0)$ & $21(53,9)$ & & $87(66,4)$ & $16(69,6)$ & $71(65,7)$ & & \\
\hline Dor nas pernas & & & & 0,022 & & & & 0,359 & 0,389 \\
\hline Sim & $55(56,1)$ & $39(66,1)$ & $16(41,0)$ & & $66(50,4)$ & $14(60,9)$ & $52(48,1)$ & & \\
\hline Não & $43(43,9)$ & $20(33,1)$ & $23(59,0)$ & & $65(49,6)$ & $9(39,1)$ & $56(51,9)$ & & \\
\hline Edema & & & & 0,633 & & & & 0,144 & 0,236 \\
\hline Sim & $23(23,5)$ & $15(25,4)$ & $8(20,5)$ & & $40(30,5)$ & $10(43,5)$ & $30(27,8)$ & & \\
\hline Não & $75(76,5)$ & $44(74,6)$ & $31(79,5)$ & & $91(69,5)$ & $13(56,5)$ & $78(72,2)$ & & \\
\hline Náusea & & & & 0,645 & & & & 0,590 & 0,855 \\
\hline Sim & $5(5,1)$ & $4(6,8)$ & $1(2,6)$ & & $6(4,6)$ & 0 & $6(5,6)$ & & \\
\hline Não & $93(94,9)$ & $55(93,2)$ & $38(97,4)$ & & $125(95,4)$ & $23(100)$ & $102(94,4)$ & & \\
\hline Palpitação & & & & 0,999 & & & & 0,771 & 0,010 \\
\hline Sim & $7(7,1)$ & $4(6,9)$ & $3(7,7)$ & & $25(19,1)$ & $5(21,7)$ & $20(18,5)$ & & \\
\hline Não & $91(92,9)$ & $55(93,2)$ & $36(92,3)$ & & $106(80,9)$ & $18(78,3)$ & $88(81,5)$ & & \\
\hline Lacrimejamento & & & & 0,516 & & & & 0,407 & 0,040 \\
\hline Sim & $2(2,0)$ & $2(3,4)$ & 0 & & $11(8,4)$ & $3(13,0)$ & $8(7,4)$ & & \\
\hline Não & $96(98)$ & $57(96,6)$ & $39(100)$ & & $120(91,6)$ & $20(87,0)$ & $100(92,6)$ & & \\
\hline Diarreia & & & & - & & & & 0,999 & 0,031 \\
\hline Sim & 0 & 0 & 0 & & $6(4,6)$ & $1(4,3)$ & $5(4,7)$ & & \\
\hline Não & $98(100)$ & $59(100)$ & $39(100)$ & & $124(95,4)$ & $22(95,7)$ & $102(95,3)$ & & \\
\hline Constipação & & & & 0,298 & & & & 0,999 & 0,014 \\
\hline Sim & $4(4,1)$ & $1(1,7)$ & $3(7,7)$ & & $18(13,7)$ & $3(13,0)$ & $15(13,9)$ & & \\
\hline Não & $94 \quad(95,9)$ & $58(98,3)$ & $36(92,3)$ & & $113(86,3)$ & $20(87,0)$ & $93(86,1)$ & & \\
\hline
\end{tabular}

a- O número máximo de valores ignorados foi de 1 para a variável “diarreia” na população urbana. * valor p do teste do qui-quadrado para heterogeneidade de proporções. ${ }^{* \star}$ valor $\mathrm{p}$ do teste exato de Fisher para heterogeneidade de proporções. 
Ao considerar o uso de agrotóxicos pode ser observado que os sintomas de boca seca, visão alterada e dor nas pernas foram mais prevalentes em idosos residentes na zona rural que os utilizam em relação aos que não os utilizam, $(25,4 \%$ vs $0 ; \mathrm{p}=0,000 ; 35,6 \%$ vs $15,4 \% ; \mathrm{p}=0,038$; $66,1 \%$ vs $41,0 \%$; $p=0,022$ ), respectivamente. Por outro lado, o relato de esquecimento foi mais prevalente nos idosos moradores na zona rural que não utilizam agrotóxicos (46,1\% vs 22,0\%; p $=0,015$ ).

A Tabela 4 descreve as características da utilização de agrotóxicos considerando somente a população de idosos que os utiliza (35,8\%). Para a maioria destes idosos a principal atividade é a agricultura $(84,1 \%), 41,5 \%$ trabalha com poli- cultura e 29,3\% cultiva somente arroz. Relacionando o tipo de agrotóxicos utilizados na lavoura, houve predomínio na utilização de organofosforados $(37,8 \%)$, sendo que um quarto da amostra não soube informar qual agrotóxico utiliza. O mesmo ocorreu para a identificação da faixa do produto utilizado conforme sua toxicidade, onde $84,1 \%$ não informaram e 7,3\% utilizam produtos com faixa vermelha, ou seja, extremamente tóxico.

Quanto ao preparo do agrotóxico a ser utilizado, foi a própria pessoa para $24,4 \%$ da amostra, $92,7 \%$ do agrotóxico é dissolvido/preparado em água, o preparo ocorre em $84,1 \%$ dos casos no local da aplicação e o material utilizado para o preparo não é utilizado com outra finalidade

Tabela 4. Características da utilização de agrotóxicos na população estudada $(\mathrm{n}=82)$.

\begin{tabular}{|c|c|c|c|c|c|}
\hline Características & $\mathbf{N}$ & $\%$ & Características & $\mathbf{N}$ & $\%$ \\
\hline Principal produção & & & Local de preparo & & 73 \\
\hline Agricultura & 69 & 84,1 & No pátio da casa & $\begin{array}{r}6 \\
69\end{array}$ & $\begin{array}{r}1,3 \\
84,1\end{array}$ \\
\hline Pecuária & 5 & 6,1 & No local de aplicação & 69 & $\begin{array}{r}84,1 \\
4.9\end{array}$ \\
\hline Agricultura e pecuária & 4 & 4,9 & Outro & 4 & $\begin{array}{l}4,9 \\
37\end{array}$ \\
\hline Não informado & 4 & 4,9 & Não informado & 3 & 3,7 \\
\hline Tipo de plantação & & & Material de preparo usado & & \\
\hline Milho & 1 & 1,2 & para outros fins & & \\
\hline Fumo & 11 & 13,4 & Não & $\begin{array}{r}79 \\
3\end{array}$ & $\begin{array}{r}96,3 \\
3,7\end{array}$ \\
\hline Soja & 3 & 3,7 & Não respondeu & 3 & 3,7 \\
\hline Arroz & 24 & 29,3 & Forma de aplicação & & \\
\hline Consumo familiar & 9 & 11,0 & A favor do vento & 22 & 26,8 \\
\hline Policultura & 34 & 41,5 & Na ausência do vento & 48 & 58,5 \\
\hline Tipo de agrotóxico utilizado & & & Independente do vento & 6 & 7,3 \\
\hline Organosfosforado & 31 & 37,8 & Não informado & 6 & 7,3 \\
\hline Carbamato & 2 & 2,4 & Uso de EPIs ${ }^{* *}$ & & \\
\hline Piretróide & 2 & 2,4 & Completo & $\begin{array}{r}7 \\
70\end{array}$ & $\begin{array}{r}8,5 \\
85,4\end{array}$ \\
\hline Mais de um tipo & 23 & 28,1 & Incompleto & 70 & 85,4 \\
\hline Outros & 5 & 6,1 & Não informado & 5 & 6,1 \\
\hline Não informado & 19 & 23,2 & Período de aplicação & & \\
\hline Faixa do produto (toxicidade decrescente & & & Manhã & 12 & $\begin{array}{r}14,6 \\
0\end{array}$ \\
\hline Vermelha & 6 & 7,3 & Tarde & 8 & 9,8 \\
\hline Amarela & 4 & 4,9 & Qualquer período & 59 & 72,0 \\
\hline Azul & 1 & 1,2 & Não informado & 3 & 3,7 \\
\hline Verde & 2 & 2,4 & Conduta no descarte da embalagem & & \\
\hline Não soube informar & 69 & 84,1 & Adequada & & \\
\hline Quem prepara & & & Devolvida para empresa & 36 & 43,9 \\
\hline A própria pessoa & 20 & 24,4 & Inadequada & & \\
\hline Familiar do idoso & 4 & 4,9 & Armazenada & 5 & 6,1 \\
\hline Outra pessoa & 52 & 63,4 & Queimada & 7 & 8,5 \\
\hline Não informado & 6 & 7,3 & Depositada no subsolo & 19 & 23,2 \\
\hline Como é preparado & & & Permanece na lavoura & 6 & 7,3 \\
\hline Dissolvida em água & 76 & 92,7 & Não informado & 9 & 11,0 \\
\hline Direto & 3 & 3,7 & & & \\
\hline Não informado & 3 & 3,7 & & & \\
\hline
\end{tabular}

"EPIs = Equipamentos de Proteção Individual; $\mathrm{n}=82$ considerou apenas idosos que relataram uso de agrotóxicos. 
em 96,3\%. Diante da forma de aplicação dos agrotóxicos na lavoura, $58,5 \%$ os aplicavam na ausência do vento, 85,4\% utilizava os Equipamentos de Proteção Individual (EPI) de forma incompleta, e $72 \%$ das aplicações ocorrem em qualquer período do dia. Em relação à conduta no descarte das embalagens vazias dos produtos, $45,1 \%$ procedem de forma inadequada ( $\mathrm{Ta}$ bela 4$)$.

\section{Discussão}

Os dados deste estudo revelam uma prevalência elevada de exposição a agrotóxicos entre a população idosa de Cachoeira do Sul, sendo que em sua maioria permanecem residindo na zona rural. O estudo indica ainda que algumas morbidades e sintomas referidos pelos idosos foram distintos quanto à zona de moradia e uso de agrotóxicos. Observou-se que as morbidades relacionadas ao sistema nervoso central, como, insônia, ansiedade, depressão, Doença de Parkinson, Doença de Alzheimer e ainda cefaleia, foram mais prevalentes em moradores da zona urbana, enquanto o diabetes ocorreu mais em idosos residentes na zona rural. Por outro lado, a Doença de Alzheimer apresentou ainda maior prevalência para idosos da zona urbana que utilizam agrotóxicos. Quanto aos sintomas relatados verificou-se que, tosse/coriza, visão alterada, alergia/ coceira, tremor, palpitação, lacrimejamento, diarreia e constipação foram mais prevalentes em idosos moradores da zona urbana. Entretanto, para idosos que residem na zona rural, visão alterada foi mais prevalente entre aqueles que utilizam agrotóxicos. Situação semelhante ocorreu para o relato de boca seca (xerostomia) e dor nas pernas, referidos principalmente por idosos que utilizam agrotóxicos.

Os resultados deste estudo devem ser analisados com cautela, considerando que a população estudada foi amostrada por conveniência, e que o número de idosos entrevistados pode não ter sido suficiente para evidenciar diferenças nas estratificações realizadas. Entretanto, um aspecto positivo foi a elevada participação dos indivíduos na pesquisa, uma vez que as entrevistas ocorriam simultaneamente a coleta dos dados da Contagem da População - IBGE/ 2007. Este estudo utilizou delineamento transversal, que proporciona rapidez e baixo custo na realização da pesquisa; contudo, como as informações sobre exposição e desfecho são coletadas no mesmo momento, pode ser afetado por causalidade reversa. Assim, não é possível determinar a temporalidade entre o uso de agrotóxicos e a presença de morbidades e sintomas relatados, apenas a sua associação. Outro aspecto a ser destacado é que a coleta da informação sobre a presença de morbidades e sintomas foram autorreferidas pelo entrevistado. Essa forma de coleta tem se mostrado um apropriado indicador da prevalência de morbidades crônicas ${ }^{15}$ e sintomas ${ }^{16}$ sendo amplamente utilizado na literatura ${ }^{17-19}$.

Apesar disso, destaca-se que este estudo demonstrou resultados importantes, que podem subsidiar futuros estudos, como também servir de linha de base para políticas públicas, na medida em que identifica prevalências de morbidades e sintomas entre moradores de zonas distintas, incluindo ainda dados da população rural, para a qual trabalhos científicos são escassos. Também aponta para a falta de conhecimento da população estudada, quanto à exposição aos agrotóxicos, tanto direta (manipulação direta com agrotóxicos) quanto indireta (desempenho de atividades em locais vizinhos à área de manipulação), suas consequências, por efeitos tóxicos pela exposição aguda ou crônica, e forma correta de utilização.

As características da população estudada foram semelhantes ao perfil sociodemográfico da população idosa brasileira do Censo de $2000^{20} \mathrm{e}$ outras pesquisas nacionais que investigaram parâmetros comportamentais ${ }^{5,21}$. Cabe salientar, em nosso estudo observamos que indivíduos com mais de 75 anos residem preferencialmente na zona urbana, embora esta diferença não tenha sido estatisticamente significativa. Uma possível explicação para esta observação poderia ser a necessidade de assistência médica mais frequente nesta faixa etária e, em caso de viuvez, frequentemente o idoso passa a residir com seus descendentes (filhos e netos) para maior segurança e qualidade de vida.

Observou-se menor nível de escolaridade e maior prevalência de atividades agrárias em idosos moradores da zona rural. Nas décadas de 10 a 40 do século XX havia menor frequência à escola, priorizando o trabalho em detrimento da educação. Possivelmente o baixo nível de escolaridade torne os idosos mais sujeitos a exposição e, consequentemente a intoxicações por agrotóxicos ${ }^{22}$.

A proporção de idosos em relação ao consumo de tabaco e bebidas alcoólicas corroborou dados de outros estudos ${ }^{5,21}$ e não foi observada diferença entre as zonas de moradia.

A maior prevalência da utilização de agrotóxicos pela população residente na zona rural pode 
ser explicada pela elevada atuação destes na atividade agropecuária em relação aos da zona urbana. O uso destes produtos tem uma relação cultural, mas informações sobre suas consequências para a saúde e sobre seu impacto ambiental são incipientes. Atualmente, a utilização de agrotóxicos ainda é justificada pelo controle de pragas, o aumento da produção e a tradição familiar ${ }^{23}$.

Após análise das morbidades mais prevalentes, percebe-se a elevada proporção de idosos que referiram hipertensão arterial sistêmica. A estreita relação entre hipertensão arterial e envelhecimento, pode ser justificada por modificações estruturais, os grandes vasos e as arteríolas aumentam sua espessura da parede, com redução da sua luz, pois há aumento do componente colágeno e diminuição do componente elástico reduzindo a capacidade do funcionamento eficien$\mathrm{te}^{24}$. Além disso, com a aposentadoria, os idosos tornam-se mais sedentários e têm maior tendência a aumentar o peso corporal e consequentemente a pressão arterial ${ }^{25}$. Sabe-se que, em idosos, a pressão arterial elevada é o fator de risco mais importante para os acidentes vasculares encefálicos, eventos cardiovasculares maiores, principalmente insuficiência cardíaca e morte por qualquer causa ${ }^{24}$.

Prévio estudo demonstra associação significativa entre idosos e insônia, queixas crônicas foram encontradas em mais de $50 \%$ de adultos com 65 anos ou mais, particularmente dificuldade para iniciar o sono ${ }^{26}$. É importante ressaltar que poucos estudos longitudinais que avaliam o impacto da idade sobre as alterações de sono e, que estudos de prevalência, frequentemente não controlam a presença de variáveis confundidoras como comorbidades e uso de medicamentos. Outro fator a ser considerado é que com o envelhecimento há uma mudança fisiológica no padrão do sono, havendo tendência a um padrão matutino de ritmo sono-vigília, com um sono mais leve, de menor duração do sono REM e de episódios de despertar mais frequentes. Sobretudo, dificuldades para dormir têm sido apontadas como preditoras de mortalidade em idosos ${ }^{27}$. Outros estudos demonstraram que a frequência dos transtornos do sono aumenta com a idade e que prováveis fatores causais sejam mudanças nos padrões do sono, hipertensão arterial sistêmica, depressão, Doença de Alzheimer, Doença de Parkinson, noctúria, uso indevido de medicamentos, entre outros ${ }^{26}$.

O reumatismo é uma doença normal na idade da população estudada, pois ocorre envelhecimento do aparelho locomotor. Em pessoas ido- sas existe uma maior incidência de doenças reumáticas, como, por exemplo, a osteoartrose, a osteoporose, a polimialgia reumática, as doenças da coluna, as deficiências de vitamina $\mathrm{D}$, entre outras $^{28}$.

Quanto à depressão, tem sido sugerido que mesmo os sintomas subliminares são frequentemente associados com significantes alterações psicossociais $^{29}$. No Brasil, a prevalência de depressão entre as pessoas idosas varia de $4,7 \%$ a $36,8 \%$, dependendo fundamentalmente do instrumento de avaliação utilizado, dos pontos de corte e da gravidade dos sintomas ${ }^{6}$. Neste estudo as morbidades foram referidas e, portanto, esta comparabilidade deve ser olhada com cautela.

$\mathrm{Na}$ análise por zona de moradia, percebe-se maior prevalência de insônia, ansiedade, depressão, cefaleia, Doença de Parkinson e Doença de Alzheimer na população urbana. Sabe-se que idosos que residem na zona rural, além das atividades do campo, realizam mais atividades domésticas, e seus domicílios, são, geralmente, multigeracionais, o que proporciona maior convívio familiar, comparando-se com os idosos residentes na zona urbana. Sugere-se também, que essas morbidades estejam relacionadas ao estilo de vida urbano ou que os indivíduos tenham migrado do campo em busca de tratamento ou ainda, que os potenciais pacientes residentes na área rural não tenham sido identificados.

Por outro lado, a maior prevalência de diabetes em moradores da zona rural, pode estar relacionada ao tipo de alimentação. Existem evidências de alterações no perfil nutricional das comunidades rurais, com maiores taxas de sobrepeso e obesidade ${ }^{30}$, fatores que favorecem o surgimento do diabetes.

Quando analisadas as morbidades e o uso de agrotóxico, a Doença de Alzheimer teve maior prevalência em indivíduos que os utilizam. A exposição a agrotóxicos pode gerar sequelas tanto sensitivas quanto motoras, além de deficiências cognitivas transitórias ou permanentes ${ }^{10}$. Estudo de coorte com indivíduos com mais de 65 anos expostos a agrotóxicos mostrou fator de risco aumentado para a Doença de Alzheimer e Doença de Parkinson ${ }^{31}$.

Quanto aos sintomas, verificou-se que dores nas pernas e na coluna lombar foram relatados por mais da metade dos idosos, independente da zona de moradia. Valores encontrados em pesquisas com idosos para dor lombar e dores nas pernas foram menores que os encontrados no presente estudo aumentando conforme a ida$\mathrm{de}^{32,33}$. É importante ressaltar que a dor é com- 
preendida como um fenômeno multifatorial, e lesão tecidual, aspectos emocionais, socioculturais e ambientais são fatores que compõem esse fenômeno ${ }^{33}$.

Em relação aos demais sintomas relatados, acredita-se que, com o envelhecimento, advêm alterações fisiológicas que podem estar associadas a patologias crônicas e degenerativas, conduzindo a déficits funcionais e declínio da mobilidade. $\mathrm{O}$ envelhecimento pode ser acompanhado por declínio progressivo da percepção, comportamento motor, cognição e funções de memória $^{34}$. Assim, as alterações de memória encontradas em idosos, podem ser consideradas um evento fisiológico, mas não necessariamente uma patologia. Neste estudo, menos de um terço dos idosos relataram o esquecimento, sem diferença entre as zonas de moradia, sugerindo a existência de um erro de recordatório, mas não de um viés de memória, o que poderia ter influenciado as respostas ao questionário.

Observando o relato dos sintomas avaliados quanto à zona de moradia, identificou-se que apesar de alguns apresentarem prevalências mais elevadas na zona urbana que na rural, não ficou estabelecido um padrão para a presença destes sintomas. Nossa hipótese inicial seria a de que idosos residentes na zona rural apresentariam mais sintomas relacionados ao uso de agrotóxicos que os da zona urbana, enquanto estes teriam maior presença de sintomas relacionados ao estilo de vida urbano. Ainda assim, percebeu-se que visão alterada e boca seca foram mais relatados pela população que utiliza agrotóxico. Este achado foi apontado também em outros estu$\operatorname{dos}^{35,36}$. Sabe-se que estes efeitos anticolinérgicos agudos são frequentes nas populações cronicamente expostas a agrotóxicos, decorrentes da estimulação ganglionar simpática ${ }^{37}$.

Quanto às características da utilização de agrotóxicos, observou-se um comportamento desfavorável à saúde, sugerindo falta de conhecimento da importância do uso completo de EPI, modo adequado de descarte das embalagens e forma de aplicação dos produtos. A ausência de uso de EPI aumenta 535\% as chances de intoxicação ${ }^{38}$. A falta de conhecimento dos malefícios dos produtos químicos e a crença de que acidente não ocorrerá, faz com que pouquíssimos indivíduos tenham consciência da importância do seu uso ${ }^{38,39}$. Também se observa a alta prevalência de conduta inadequada no descarte das embalagens dos agrotóxicos corroborando com outro estudo prévio que sugere que esta prática como um descaso com o meio ambiente ${ }^{36}$, pois contaminará o solo e as águas subterrâneas, muitas vezes utilizadas pelas famílias dos próprios agricultores e pela população em geral. É possível sugerir que a baixa escolaridade possa determinar a dificuldade de compreender informações sobre a adequada utilização de agrotóxicos e o impacto do uso dos mesmos à saúde e ao meio ambiente. Ressalta-se ainda que por existirem interesses por parte dos fabricantes em comercializar esses produtos, por vezes omitem informações importantes e não elucidam sobre alternativas a seu emprego.

\section{Conclusão}

Os resultados obtidos representam uma contribuição significativa, diante da carência de publicações que revelem a forma como as pessoas expõem-se a agrotóxicos, principalmente considerando a faixa etária estudada, que apresenta uma maior probabilidade de ter sido exposta a agrotóxicos por longo tempo. Salienta-se que o delineamento transversal não determina riscos absolutos, o que torna importante a realização de estudos longitudinais para estabelecer a relação de causalidade. Além disso, é importante salientar a necessidade de desenvolvimento de programas de saúde pública que auxiliem na promoção de saúde do idoso, e considerar a possível exposição desta população a agentes ambientais, incluindo os agrotóxicos. Também se fazem necessários, esclarecimentos tanto dos potenciais riscos aos indivíduos que manuseiam esses produtos, quantos indivíduos que são expostos indiretamente. 


\section{Colaboradores}

EF Silva trabalhou na concepção, no delineamento e redação do artigo. VMV Paniz trabalhou na análise e interpretação dos dados, redação do artigo e aprovação final da versão a ser publicada. G Laste trabalhou na redação do artigo. ILS Torres trabalhou na concepção, no delineamento, redação do artigo e aprovação final da versão a ser publicada.

\section{Agradecimentos}

Ao Programa de Pós Graduação Ambiente e Desenvolvimento do Centro Universitário Univates - Lajeado (RS) pelo apoio financeiro que possibilitou a realização deste estudo.

\section{Referências}

1. Paskulin LMG, Vianna LAC. Perfil de idosos de Porto Alegre. Rev Saude Publica 2007; 41(5):757-768.

2. Dias Júnior CS, Costab CS, Lacerda MA. O envelhecimento da população brasileira: uma análise de conteúdo das páginas da REBEP. Rev Bras Geriatr Gerontol 2006; 9(2):7-24.

3. Chaimowicz F. Epidemiologia e o envelhecimento no Brasil. In: Freitas EV, Py L, Cançado FAX, Gorzoni ML, Doll J, organizadores. Tratado de geriatria e gerontologia. 2a Edição. Rio de Janeiro: Guanabara Koogan; 2006.

4. Instituto Brasileiro de Geografia e Estatística (IBGE). Evolução da razão de dependência da população, total e por grupos de idade específicos e indice de envelhecimento - Brasil - 1940-2050. Rio de Janeiro: Indicadores sociodemográficos e de saúde no Brasil; 2009.

5. Peixoto SV, Firmo JA, Lima-Costa M. Condições de saúde e tabagismo entre idosos residentes em duas comunidades brasileiras (Projetos Bambuí e Belo Horizonte). Cad Saude Publica 2006; 22(9):1925-1934.

6. Brasil. Ministério da Saúde (MS). Cadernos de Atenção Básica: envelhecimento e saúde da pessoa idosa. Brasília: MS; 2006.

7. Jobim PFC, Nunes LN, Giugliani R, Cruz IBM. Existe uma associação entre mortalidade por câncer e uso de agrotóxicos? Uma contribuição ao debate. Cien Saude Colet 2010; 15(1):277-288.

8. Naidoo S, London L, Rother H-A, Burdorf A, Naidoo Kromhout H. Pesticide safety training and practices in women working in small-scale agriculture in South Africa. Occup Environ Med 2010; 67(12):823828.

9. Waggoner JK, Kullman GJ, Henneberger PK, Umbach DM, Blair A, Alavanja MCR, Kamel F, Lynch CF, Knott C, London SJ, Hines CJ, Thomas KW, Sandle DPr, Lubin JH, Freeman LEB, Hoppin JA. Mortality in the Agricultural Health Study, 19932007. Am J Epidemiol 2011; 173(1):71-83.

10. Issa Y, Sham'a FA, Nijem K, Bjertness E, Kristensen P. Pesticide use and opportunities of exposure among farmers and their families: cross-sectional studies 1998-2006 from Hebron governorate, ccupied Palestinian territory. Environ Health 2010; 9:63.

11. Araoud M, Neffeti F, Douki W, Najjar MF, Kenani A. Paraoxonase 1 correlates with butyrylcholinesterase and gamma glutamyl transferase in workers chronically exposed to pesticides. J Occup Health 2010; 52(6):383-388.

12. Instituto Brasileiro de Geografia e Estatística (IBGE). Cidades. [acessado 2011 jul 05]. Disponível em: http:/ /www.ibge.gov.br/cidadesat/topwindow.htm?1/

13. Souza A, Medeiros AR, Souza AC, Wink M, Siqueira IR, Ferreira MBC, Fernandes L, Hidalgo MPL, Torres ILS. Avaliação do impacto da exposição a agrotóxicos sobre a saúde de população rural: Vale do Taquari (RS, Brasil). Cien Saude Colet 2011; 16(8):3519-3528.

14. Brasil. Ministério da Saúde (MS). Conselho Nacional de Saúde. Resolução no 196 de 10 de outubro de 1996. Diretrizes e Normas Regulamentadoras de Pesquisas Envolvendo Seres Humanos. Diário Oficial da União 1996; out 16. 
15. Lima-Costa MF, Peixoto SV, Firmo JOA. Validade da hipertensão arterial auto-referida e seus determinantes (projeto Bambuí). Rev Saude Publica 2004; 38(5):637-642.

16. Mendoza-Sassi R, Beria JU, Fiori N, Bortolotto A. Prevalência de sinais e sintomas, fatores sociodemográficos associados e atitude frente aos sintomas em um centro urbano no Sul do Brasil. Rev Panam Salud Publica 2006; 20(1):22-28.

17. Arrais PSD, Brito LL, Barreto ML, Coelho HLL. Prevalência e fatores determinantes do consumo de medicamentos no Município de Fortaleza, Ceará, Brasil. Cad Saude Publica 2005; 21(6):1737-1746.

18. Almeida AAL, Bonfante HLM, Moreira RO, Arbex AK, Souza GS, Maciel LG, Godinho RR, Giannini G. Perfil epidemiológico do diabetes mellitus autoreferido em uma zona urbana de Juiz de Fora, Minas Gerais. Arq Bras Endocrinol Metab 1999; 43(3):199-204.

19. Faria NMX, Facchini LA, Fassa AG, Tomasi E. Estudo transversal sobre saúde mental de agricultores da Serra Gaúcha (Brasil). Rev Saude Publica 1999; 33(4):391-400.

20. Instituto Brasileiro de Geografia e Estatística (IBGE). Censos Demográficos. [acessado 2011 jul 02]. Disponível em: http://www.ibge.gov.br/home/estatistica/ populacao/default_censo_2000.shtm/

21. Barros MBA, Marín-Leó L, Oliveira HB, Dalgalarrondo P, Botega NJ. Perfil do consumo de bebidas alcoólicas: diferenças sociais e demográficas no município de Campinas, estado de São Paulo, Brasil 2003. Epidemiol Serv Saúde 2008; 17(4):259-270.

22. Rothlein J, Rohlman D, Lasarev M, Phillips J, Muniz J, McCauley L. Organophosphate pesticide exposure and neurobehavioral performance in agricultural and nonagricultural hispanic workers. Environ Health Perspect 2006; 114(5):691-696.

23. Jacobson LSV, Hacon SS, Alvarenga L, Goldstein RA, Gums C, Buss DF, Leda LR. Comunidade pomerana e uso de agrotóxicos: uma realidade pouco conhecida. Cien Saude Colet 2009; 14(6):2239-2249.

24. Gazoni FM Braga ILS, Guimarães HP, Lopes RD. Hipertensão sistólica no idoso. Rev Bras Hipertens 2009; 16(1):34-37.

25. Amado TC, Arruda IK. Hipertensão arterial no idoso e fatores de risco associados. Rev Bras Nutr Clin 2004; 19(2):94-99.

26. Quinhones MS, Gomes MM. Sono no envelhecimento normal e patológico: aspectos clínicos e fisiopatológicos. Rev Bras Neurol 2011; 47(1):31-42.

27. Dew MA, Hoch CC, Buysse DJ, Monk TH, Begley AE, Houck PR, Hall M, Kupfer DJ, Reynolds CF. Healthy older adults' sleep predicts all-cause mortality at 4 to 19 years of follow-up. Psychosom Med 2003; 65(1):63-73

28. Holick MF. Vitamin D deficiency. $N$ Engl J Med 2007; 357(3):266-281

29. Ayotte BJ, Potter GJ, Williams HJ, Steffens DC, Bosworth HB. The moderating role of personality factors in the relationship between depression and neuropsychological functioning among older adults. Int J Geriatr Psychiatr 2009; 24(9):1010-1019.
30. Mendes LL, Gazzinelli A, Velásquez-Meléndez G. Fatores associados à resistência à insulina em populações rurais. Arq Bras Endocrinol Metab 2009; 53(3):332-339.

31. Hayden KM, Norton MC, Darcey D, Ostbye T, Zandi PP, Breitner JCS, Welsh-Bohmer KA. Occupational exposure to pesticides increases the risk of incident AD. Neurology 2010; 74(19):1524-1530.

32. Reis LA, Mascarenhas CHM, Marinho-Filho LEN, Borges PS. Lombalgia na terceira idade: distribuição e prevalência na Clínica Escola de Fisioterapia da Universidade Estadual do Sudoeste da Bahia. Rev Bras Geriatr Gerontol 2008; 11(1):93-103.

33. Dellaroza MSG, Pimenta CAM, Matsu T. Prevalência e caracterização da dor crônica em idosos não institucionalizados. Cad Saude Publica 2007; 23(5): 1151-1160.

34. Kattenstroth J-C, Kolankowska I, Kalisch T, Dinse HR. Superior sensory, motor, and cognitive performance in elderly individuals with multi-year dancing activities. Front Ag Neurosc 2010; 2:31.

35. Ávila RA, Rezende DMLC, Resende ILCR, Resende GAA. Trabalho rural e agrotóxicos: estudo de caso na microbacia do córrego Água Limpa, município de Campos Altos, Minas Gerais. Pesticidas: r. ecotoxicol. e meio ambiente 2009; 19:73-80.

36. Delgado IF, Paumgartten FR. Intoxicações e uso de pesticidas por agricultores do município de Paty do Alferes, Rio de Janeiro, Brasil. Cad Saude Publica 2004; 20(1):180-186.

37. Goodman LS, Brunton LL, Chabner B, Knollmann BC. Goodman \& Gilman's the Pharmacological Basis of Therapeutics. 12a Edição. New York: McGrawHill Medical; 2010.

38. Soares WL, Freitas EA, Coutinho JA. Trabalho rural e saúde: intoxicações por agrotóxicos no município de Teresópolis - RJ. Rev Econ Sociol Rural 2005 43:685-701.

39. Araújo AJ, Lima JS, Moreira JC, Jacob SC, Soares MO, Monteiro MC, Amaral AM, Kubota A, Meyer A, Cosenza CAN, Neves C, Markowitz S. Exposição múltipla a agrotóxicos e efeitos à saúde: estudo transversal em amostra de 102 trabalhadores rurais, Nova Friburgo, RJ. Cien Saude Colet 2007; 12(1):1115-1130.

Artigo apresentado em 12/10/2011

Aprovado em 20/10/2011

Versão final apresentada em 04/11/2011 